\title{
A new method for chlorhexidine (CHX) determination: CHX release after application of differently concentrated CHX-containing preparations on artificial fissures
}

\author{
Thomas Attin • Thaer Abouassi • Klaus Becker • \\ Annette Wiegand • Malgorzata Roos • Rengin Attin
}

Received: 17 August 2007 / Accepted: 5 November 2007 / Published online: 4 December 2007

(C) Springer-Verlag 2007

\begin{abstract}
Aims of the study were (1) to establish a method for quantification of chlorhexidine (CHX) in small volumes and (2) to determine CHX release from differently concentrated CHX-containing preparations, varnishes, and a CHX gel applied on artificial fissures. CHX determination was conducted in a microplate reader using polystyrene wells. The reduced intensity of fluorescence of the microplates was used for CHX quantification. For verification of the technique, intra- and inter-assay coefficients of variation were
\end{abstract}

T. Attin $\cdot$ K. Becker $\cdot$ A. Wiegand

Clinic for Preventive Dentistry, Periodontology and Cariology, University Zurich,

Zurich, Switzerland

T. Attin

Department of Operative Dentistry,

Preventive Dentistry and Periodontology,

University of Göttingen,

Göttingen, Germany

T. Abouassi

Department of Operative Dentistry and Periodontology,

University of Freiburg,

Freiburg, Germany

M. Roos

Biostatistics Unit, Institute of Social and Preventive Medicine,

University Zurich,

Zurich, Switzerland

T. Attin $(\bowtie)$

University Dental School,

Plattenstr. 11,

CH-8032 Zurich, Switzerland

e-mail: thomas.attin@zzmk.uzh.ch

Present address:

R. Attin

Zurich, Switzerland calculated for graded series of CHX concentrations, and the lower limit of quantification (LLOQ) was determined. Additionally, artificial fissures were prepared in 50 bovine enamel samples, divided into five groups (A-E, $n=10)$ and stored in distilled water (7 days); A: CHX-varnish EC40; B: CHX-varnish Cervitec; C: CHX-gel Chlorhexamed; D: negative control, no CHX application; and E: CXH-diacetate standard $(\mathrm{E} 1, n=5)$ or CHX-digluconate $(\mathrm{E} 2, n=5)$ in the solution. The specimens were brushed daily, and CHX in the solution was measured. The method showed intra- and inter-assay coefficients of variation of $<10$ and $<20 \%$, respectively; LLOQ was $0.91-1.22 \mathrm{nmol} /$ well. The cumulative CHX release (mean \pm SD) during the 7 days was: EC40 $(217.2 \pm 41.8 \mathrm{nmol})$, CHX-gel $(31.3 \pm 8.5 \mathrm{nmol})$, Cervitec $(18.6 \pm 1.7 \mathrm{nmol})$. Groups A-C revealed a significantly higher CHX release than group D and a continuous CHXrelease with the highest increase from day 0 to 7 for EC40 and the lowest for Chlorhexamed. The new method is a reliable tool to quantify CHX in small volumes. Both tested varnishes demonstrate prolonged and higher CHX release from artificial fissures than the CHX-gel tested.

Keywords Varnish · Chlorhexidine - EC40 - Cervitec . Chlorhexamed gel $\cdot$ CHX determination

\section{Introduction}

Chlorhexidine (CHX) is considered as one of the most popular agents to reduce the risk of developing new caries lesions $[21,26,32]$. One reason for this estimation is the broad antiseptic property of CHX against a wide variety of gram-negative and gram-positive organisms. In high concentrations, $\mathrm{CHX}$ is bactericidal via destruction of the cell membrane [16]. The antimicrobial activity of CHX is due 
to the positively charged parts of the CHX molecule, which react with the phosphate groups of lipopolysaccharides in the bacterial cell wall. At lower concentrations, $\mathrm{CHX}$ has bacteriostatic properties. For use in the oral cavity, $\mathrm{CHX}$ is available and effective in different delivery systems, such as sprays, mouthwashes, CHX-containing glass ionomer cement gels, chips, and varnishes [1, 4, 5, 12, 19, 25, 27, 33]. Varnishes are usually applied in the dental office and act as a kind of slow-releasing device, thus resulting in a prolonged intraoral CHX availability as compared to mouthwashes and gels.

Chlorhexidine varnishes might be used for prevention of fissure caries, although the results of studies in high-risk caries patients are inconclusive $[38,39]$. Especially in the time period shortly after eruption of the teeth, it is often difficult to apply adhesively attached fissure sealants due to insufficient moisture control. In this phase, CHX varnishes might help to fight bacterial inoculation of the fissure systems and to postpone application of fissure sealants to a time point when moisture control could be guaranteed.

However, application of CHX varnish onto fissures resulted in contradictory results in terms of fissure caries reduction in studies running over periods from 9 months to 3 years [39]. On the one hand, some recent split-mouth controlled studies and randomized clinical trials of various research groups $[3,7,8,10,23]$ proved the CHX varnish Cervitec (1\% CHX and 1\% thymol) being effective to significantly reduce the caries incidence in molar fissures in groups of children and adolescents. In contrast, in a randomized controlled study using the $40 \%$ CHX varnish EC40, no statistically significant benefit was recorded in the group treated with the varnish as compared to the placebo group [15].

CHX has the ability to adsorb onto tooth surfaces and oral mucosa, with a slow release later on. When applied onto fissures, it is additionally possible that remnants of the applied varnish will retain even after mechanical impact, such as mastication and toothbrushing. These varnish remnants will allow for prolonged and elevated CHX levels in the fissure systems. Previous studies using Cervitec could show that this varnish is able to release $\mathrm{CHX}$ for as long as 3 months with the majority of release during the first 4 weeks of storage [20]. In this study, the varnish was applied on microscope glass slides $(2 \times 7.5 \times$ $2.5 \mathrm{~cm}$ ) resulting in a measurable amount of CHX release from the varnish into the $50-\mathrm{ml}$ sample solution. This experimental setup allowed for determination of the CHX release with a common ultraviolet (UV) spectrophotometer. Using UV absorption for CHX determination needs both expensive quartz cuvettes and a sample volume of at least $3 \mathrm{ml}$. Additionally, application of varnishes on glass plates does not simulate intraoral conditions with mechanical impact such as toothbrushing. When applied onto the fissure system of a tooth, which is regularly brushed, only small quantities of CHX are expected to be released into the solution used to store the respective specimens. Thus, to increase the concentration in the solution, the specimens should be stored in small sample volumes. However, using the above-mentioned UV-absorption method as done in various studies $[20,28,31]$, it is difficult to measure reliably such low concentrations in small sample volumes. More sensitive methods, such as high-performance liquid chromatography or ion mobility spectrometry have also been described for CHX determination [9, 11, 37, 40]. However, these approaches do need extensive sample preparation and expensive devices and does not allow remeasuring in the same sample solution, which is needed for monitoring the release over a certain period of time. Additionally, colorimetric methods using different markers for CHX, such as eosin, bromthymolblue, methylene orange and bromchresol green, have been described for CHX determination [2, 13, 18, 30, 42]. Disadvantages of these methods are either the low sensitivity or the fact that the CHX has to be extracted in the sample solution using chloroform, not allowing monitoring in the same sample solution over a period of time.

It would be useful for clinical practice to have information about the characteristics of CHX release from varnishes applied onto tooth surfaces and fissure systems.

Thus, the aims of the study were:

1) To develop and to verify an unexpensive, sensitive method for determination of low CHX concentrations in small sample volumes

2) To determine the period of time of chlorhexidine release from CHX varnishes applied on artificial fissures subjected to toothbrushing

\section{Materials and methods}

Principle of the method to quantify CHX in small sample volumes

The following principle for quantification of CHX in small volumes was developed in the laboratory of the authors. The determination of CHX was conducted using polystyrene flat-bottom microplate wells (Sarstedt, Nümbrecht, Germany) in a microplate reader (SpectraMax M2, Molecular Devices, Ismaning/Munich, Germany). When excited with $280 \mathrm{~nm}$ from the top, the bottom of the polysterene plate shows fluorescence, resulting in light emittance with a wavelength of $360-380 \mathrm{~nm}$. Chlorhexidine is able to absorb light with the wavelength of $280 \mathrm{~nm}$ during the passage but not the emitted light, resulting in a decreased intensity of 
the fluorescence of the polystyrene (measured as RFU, relative fluorescence intensity; Fig. 1). When calculating the relation between the RFU of a blank well and a well containing a CHX solution excited at $280 \mathrm{~nm}$, the best results are achieved at emitted fluorescence of $370 \mathrm{~nm}$. This finding indicates the high sensitivity to determine $\mathrm{CHX}$ when measuring intensity of emitted fluorescence at $370 \mathrm{~nm}$ (Fig. 1).

In a first step, the unfilled polystyrene wells of the microplates were excited with $280 \mathrm{~nm}$, and the emitted fluorescence was recorded at $370 \mathrm{~nm}$ to determine the blank value. Then, $370 \mu \mathrm{l}$ each $(1 \mathrm{~cm}$ height in a well) of a standard dilution series with $0,7,14,28,56,111$, and $223 \mu \mathrm{mol} / \mathrm{l}$ CHX was applied into the wells to create a standard calibration curve. The final content of CHX in the respective wells corresponded to $0,2.6,5.2,10.3,20.6$, 41.2, and $82.4 \mathrm{nmol}$ CHX per well. The standard calibration curve was fitted with polygonal interpolation. Relative fluorescence was expressed as percentage of the blank value and was recorded. By means of the standard calibration curve, the absolute quantity of CHX (nmol) per well is determined. Since the CHX regimes later used in the study contained different CHX formulations, standard calibration curves were recorded for both CHX-diacetate and CHX-digluconate. No differences were observed for these two compounds with respect to the calibration curves recorded (Fig. 2). Owing to that finding, CHX-diacetate was used in the study later on for measuring the CHX release from the CHX-treated specimens.

Validation of the method

Precision, reproducibility and lower limit of quantification (LLOQ) were checked according to the guidance for bioanalytical method validation recently described by Shah et al. $[34,35]$. The measurements were performed at room temperature of $25^{\circ} \mathrm{C}$. The experiments were run with ten repeats in series using graded dilution series of $\mathrm{CHX}$ diacatate from 0.7 to $78.4 \mathrm{nmol} /$ well. Either 100,200 or $370 \mu$ were pipetted into each well.

Intra-assay coefficient of variation of CHX recovery was calculated for assessing precision of the test since it considers both distribution of the data and slope of the calibration curve. Threshold for acceptable precision was set at a coefficient of variation of $<10 \%$. For example, an intra-assay coefficient of variation of $10 \%$ would mean that a CHX concentration of $100 \mu \mathrm{mol} / \mathrm{l}$ in a respective solution would yield a reading in a range of $90-110 \mu \mathrm{mol} / \mathrm{l}$.

Reproducibility was checked by calculating the interassay coefficient of variation. Therefore, for each solution,
Fig. 1 Above, schematic drawing of the principle of the CHX-determination (right) measuring the reduced fluorescence of the polysterene plates due to absorbance of the excitation light by the CHX in the solution (left). Below, intensity (relative fluorescence units, RFU) of emission wavelengths $(\mathrm{nm})$ determining a solution without (no CHX) and with $0.01 \%$ CHX in the polysterene plates excited with $280 \mathrm{~nm}$. Additionally, the relations between the fluorescence intensity measured for the solution without and with $0.01 \% \mathrm{CHX}$ are presented
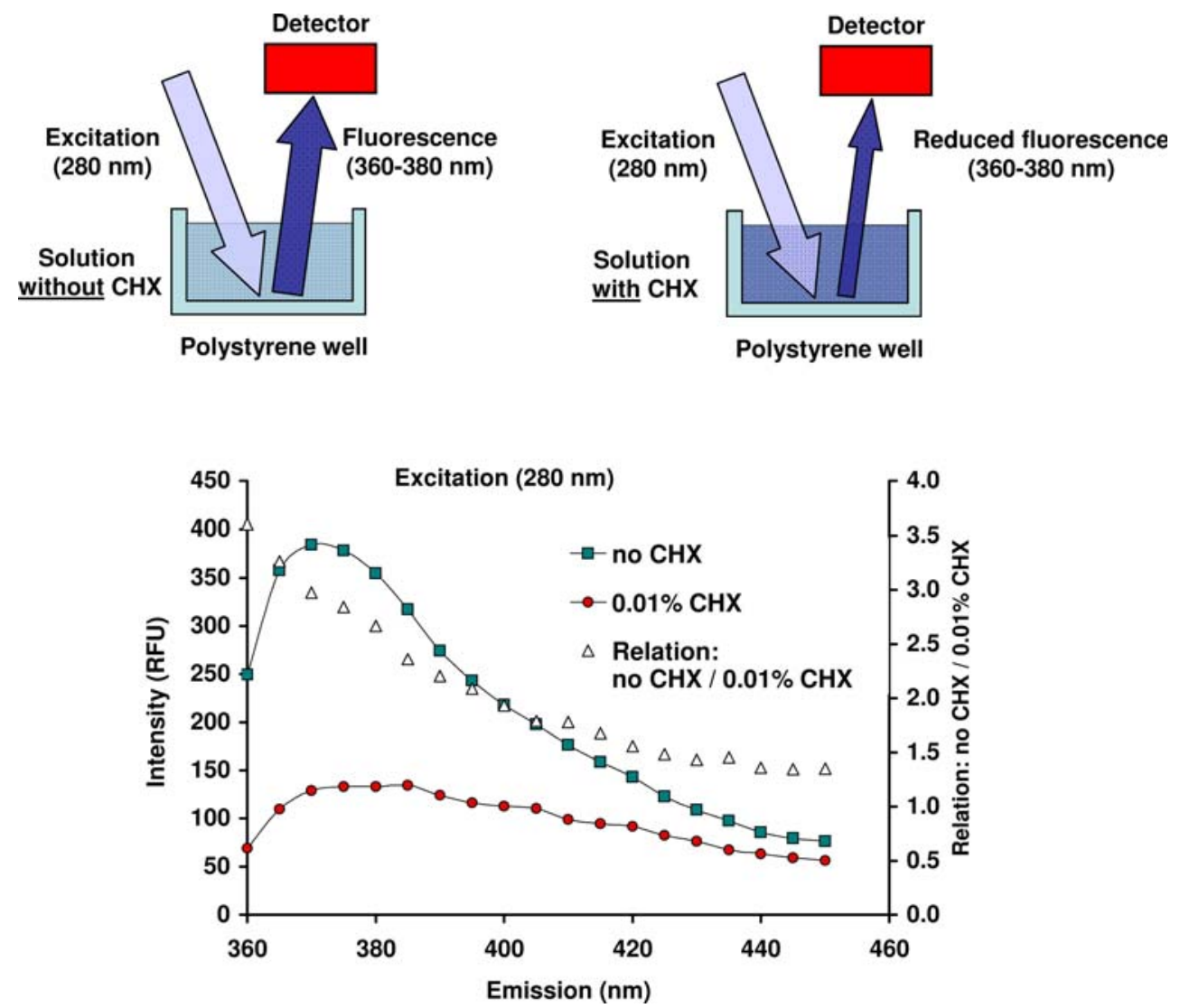


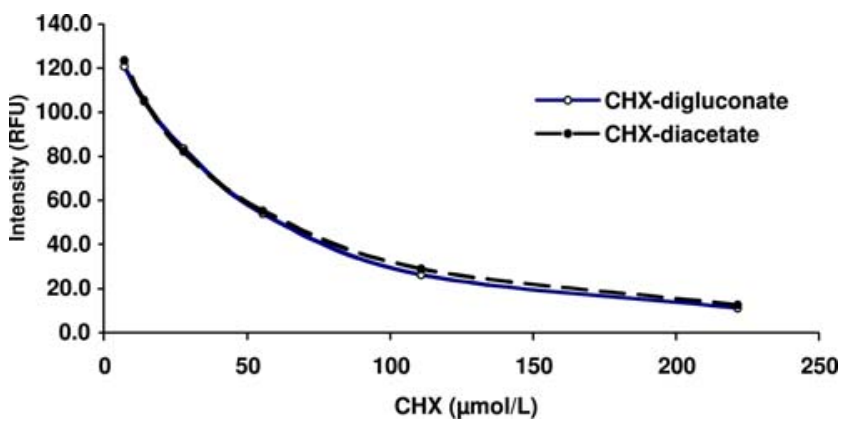

Fig. 2 Mean intensity (relative fluorescence units, RFU) of differently concentrated solutions of CHX-digluconate and CHX-diacatate

ten calibration curves were constructed, and recovery of CHX and inter-assay coefficient of variation was calculated. Threshold for acceptable reproducibility was set at an inter-assay coefficient of variation of $<20 \%$. For example, an inter-assay coefficient of variation of $<20 \%$ would mean that using different calibration curves a CHX concentration of $100 \mu \mathrm{mol} / 1$ in a respective solution would yield a result of $80-120 \mu \mathrm{mol} / 1$.

Lower limits of quantification (nmol/well) for the respective solutions were calculated as CHX concentration at the particular point on the calibration curve presenting the following: (mean value of blank fluorescence) minus $(5 \times$ standard deviation). As a threshold, the lowest standard on the calibration curve $(2.6 \mathrm{nmol} /$ well $)$ should be higher than the LLOQ [34, 35].

\section{Preparation of enamel samples}

Fifty bovine central lower incisors were used in the study. After extraction, the teeth had been stored in $0.1 \%$ thymol until use. Cylindrical samples (5 $\mathrm{mm}$ in diameter) were prepared from the labial surface with a diamond-coated trephine drill (Geb. Brasseler/Komet, Lemgo, Germany). The cylinders were then embedded in acrylic resin (Technovit 4071 ${ }^{\circledR}$, Kulzer, Wehrheim, Germany). Subsequently, the specimens were ground flat with water-cooled carborundum discs (500-4,000 grit; Water Proof Silicon Carbide Paper, Struers, Erkrath, Germany). Grinding and polishing resulted in approximately $100 \mu \mathrm{m}$ of the enamel being removed. The amount of abraded enamel was controlled with a micrometer (Digimatic; Mitutoyo-Meßgeräte, Leonberg, Germany).

Using a diamond-coated disc (Komet), grooves $(0.5 \mathrm{~mm}$ in depth and $0.2 \mathrm{~mm}$ in width) were prepared on the polished surface under constant water-cooling. The grooves were arranged in the form of four crosses. A special device designed for this purpose was utilized to guarantee standardized preparation of the grooves. After cleaning the artificial fissures with water and air-drying, the samples were divided into five groups (A-E) of ten samples each:

- Group A (CHX varnish EC40)
The varnish EC40 (explore, NL-6501, Nijmegen, Netherlands) contains $40 \%$ CHX-diacetate in a sandarac resin base, dissolved in water-free alcohol and is packed in glass carpules. The varnish was applied onto the enamel surface of the samples in a thin layer of about $1 \mathrm{~mm}$ using a syringe with a blunt needle.

After setting of the varnish for $10 \mathrm{~min}$, gross material excess was removed with a probe as instructed by the manufacturer. Subsequently, the surfaces of the enamel samples were brushed with 40 strokes in an automatic brushing machine [41]. The toothbrush Oral-B classic soft (Procter \& Gamble, Schwalbach am Taunus, Germany) with a load of $200 \mathrm{~g}$ was used in a toothpaste slurry consisting of the dentifrice elmex (Gaba, Münchenstein, Switzerland) dissolved in distilled water in a ratio of $1 \mathrm{~g}: 3 \mathrm{ml}$. The samples were then stored in $700 \mu \mathrm{l}$ distilled water in closed polyethylene containers for 7 days and brushed every day.

- Group B (CHX varnish Cervitec)

Cervitec (Ivoclar Vivadent, Schaan, Liechtenstein) contains 1\% CHX-diacetate and 1\% thymol dissolved in ethanol, ethyl acetate and a polymer (polyvinyl butyral), and is packed in glass vials $(1.5 \mathrm{ml})$. The user's instruction recommends the first toothbrushing not until $24 \mathrm{~h}$ after application of the varnish. The varnish was applied in two layers onto the enamel samples with a microbrush.

The samples were also transferred to a vial containing $700 \mu \mathrm{l}$ of distilled water. The first brushing was conducted after a period of $24 \mathrm{~h}$ storage. Thereafter, the samples were brushed every day as described for group A.

- Group C (CHX gel Chlorhexamed)

The Chlorhexamed gel (GlaxoSmithKline, Bühl, Germany) contains $1 \%$ CHX-digluconate $1 \%$. The gel was applied with a microbrush onto the enamel surfaces in a layer of about $1 \mathrm{~mm}$ thickness. First brushing of the samples was conducted after the application of the gel, before transferral to the storage media. Thereafter, the samples were brushed every day as described for group A.

- Group D (negative controls)

Neither gel nor varnish was applied on the enamel samples. These samples were brushed and were stored in $700 \mu \mathrm{l}$ distilled water also.

\section{- Group E}

No application of CHX onto the enamel surface was done, like in group D. However, these samples were not brushed and were subdivided into two subgroups (E1 and E2) of five specimens each with respect to the storage media. In group E1, samples were stored in $700 \mu \mathrm{l}$ of a solution containing $55.4 \mu \mathrm{mol} / \mathrm{l}$ chlorhexidine-diacetate (i.e., $38.8 \mathrm{nmol}$ CHX-diacetate). In group E2, the storage media contained $55.7 \mu \mathrm{mol} / 1$ chlorhexidine-digluconate in the $700 \mu$ solution (i.e., $39.0 \mathrm{nmol}$ CHX-digluconate). Groups E1 and E2 were included in the study for checking adsorption of the different CHX compounds to the enamel 
surface. The values recorded for these samples were later used to calculate the net release from the different CHX preparations.

Determination of CHX in the storage media

As mentioned above, each sample was stored in $700 \mu \mathrm{l}$ distilled water for a period of 7 days. On each day, $370 \mu \mathrm{l}$ were pipetted from the solution $3 \mathrm{~h}$ after brushing and used for determination of $\mathrm{CHX}$ in the microplate reader as described above. After determination, the $370 \mu$ liquid sample was retransferred to the vials containing the enamel samples. Thus, at the end of the experiment, CHX measurement in the solution represented the cumulative CHX release over the 7-day period. The values recorded for each day were corrected by the value obtained in group $\mathrm{E}$ (adsorption to enamel surface), so that the net release of the CHX regimes was used in the statistical analysis and presentation of the data in the result section.

The determination gave the absolute amount of CHX (nmol) in the $370 \mu \mathrm{l}$ samples. With this value, the absolute amount of CHX (nmol)] released from the CHX-treated enamel samples into the $700 \mu \mathrm{l}$ storage solution was calculated.

\section{Statistical analysis}

Comparisons between the different groups regarding CHX release directly after application of the regimes were performed with one-way analysis of variance (ANOVA). The increase of CHX release over time (starting after day 0 to day 7) was conducted with a weighted one-way ANOVA and post hoc-tests with adjustments according to BonferroniDunn. The level of significance was set at $P<0.05$.

\section{Results}

Validation of the method for CHX-determination

The recovery rate of CHX measured in different volumes $(100,200$, and $370 \mu \mathrm{l})$ with $0.7-78.4 \mathrm{nmol} \mathrm{CHX}$ per well ranged from 99.1 to $100.4 \%$. In Figs. 3 and 4, the intra- and inter-assay coefficients of variation of the CHX determination with different concentrations and different volumes are given. The intra-assay coefficients of variation amounted to $<10 \%$ for most of the solutions tested except for levels below $2.8 \mathrm{nmol} /$ well in 100 or $200 \mu \mathrm{l}$ and below $3.5 \mathrm{nmol} / \mathrm{well}$ in $370 \mu \mathrm{l}$ solution. All interassay coefficients of variation were below the threshold level of $20 \%$. Lower limits of quantification for the different volumes applied to the wells of the microtiter plate

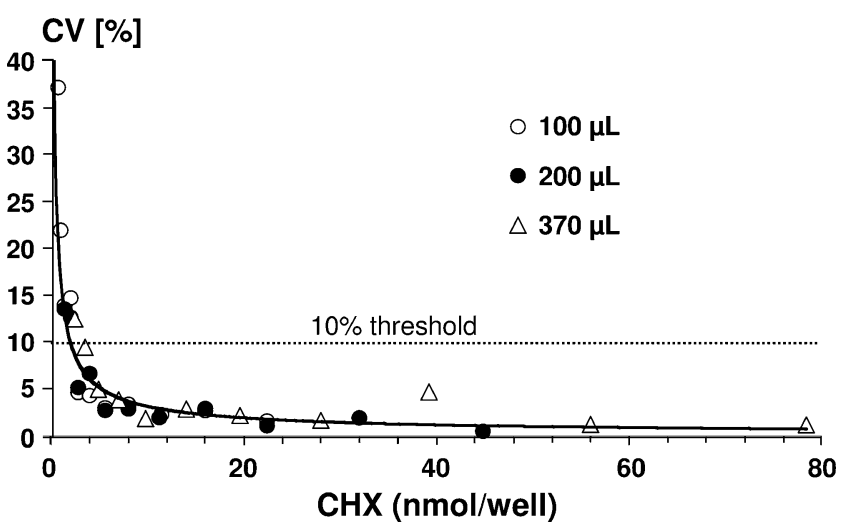

Fig. 3 Intra-assay coefficients of variation (CV\%) for different CHX amounts (nmol/well) measured in volumes of 100, 200, and $370 \mu \mathrm{l}$ in the wells of microtiter plates. The $10 \%$ threshold area is marked with a dotted line

were calculated as follows: $1.22 \mathrm{nmol} / \mathrm{well}(100 \mu \mathrm{l})$, $0.91 \mathrm{nmol} /$ well $(200 \mu \mathrm{l})$ and $1.01 \mathrm{nmol} /$ well $(370 \mu \mathrm{l})$. Thus, the requirement was fulfilled that the LLOQ should be lower than the lowest standard on the calibration curve ( $2.6 \mathrm{nmol} / \mathrm{well})$.

CHX-determinations in test and control samples

The control group did not show any measurable amounts of $\mathrm{CHX}$ in the solutions at any time during the experiment. The samples of group E showed a loss of both CHXdiacatate (E1) and CHX-digluconate (E2) from the solutions during the 7-day period. From the $38.8 \mathrm{nmol}$ of CHX-diacetate and $39.0 \mathrm{nmol}$ CHX-digluconate in the solutions, only $22.9 \pm 4.9 \mathrm{nmol}$ (E1) and $22.4 \pm 8.5 \mathrm{nmol}$ (E2) could be recovered on day 7 . This finding indicated that about $41-42 \%$ of the CHX from these solutions were adsorbed on the enamel surfaces during the 7-day period.

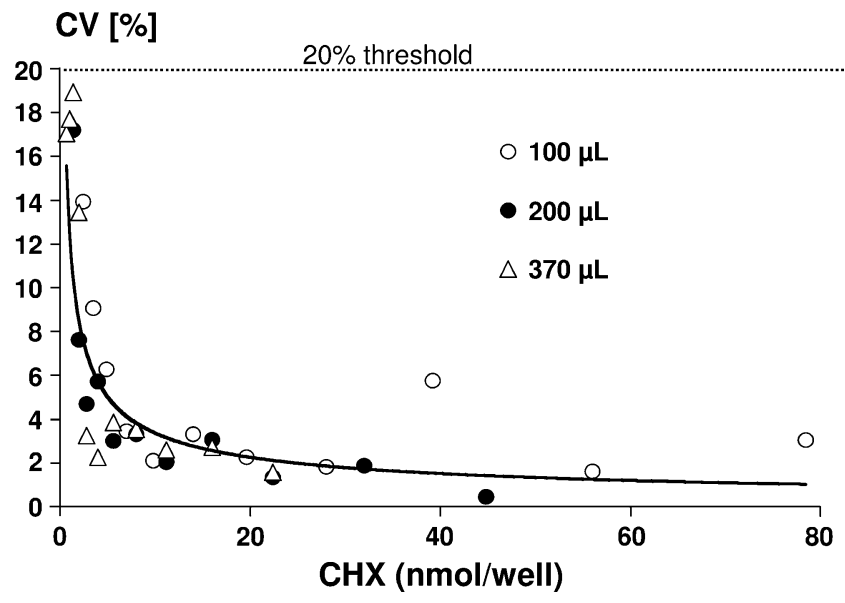

Fig. 4 Inter-assay coefficients of variation (CV\%) for different $\mathrm{CHX}$ amounts (nmol/well) determined in volumes of 100, 200, and $370 \mu 1$ in the wells of microtiter plates. The $20 \%$ threshold area is marked with a dotted line 
In Fig. 5, the cumulative CHX net release from the different regimes are given. Due to the different CHX concentrations of the regimes, significantly different amounts of CHX were released especially directly after application. The release directly after application at day 0 was therefore statistically significantly highest for EC40 as compared to the two other treatments $(P<0.0001)$. The varnish Cervitec released the significantly lowest amount of $\mathrm{CHX}(P<$ 0.0001 ). For better comparison of the CHX release over time, the cumulative CHX release after day 0 to 7 was additionally calculated. This means that the values of day 1 to 7 were added. All test groups showed a continuous release of CHX after day 0 to 7 with the statistically highest increase for EC40 and the lowest for Chlorhexamed. The increases from day 0 to 7 and the $P$ values of the increases were as follows: EC40 $(174.3 \pm 26 \mathrm{nmol} ; P<0.0001)$, Cervitec $(17.9 \pm 1.6 \mathrm{nmol} ; P<0.0001)$ and Chlorhexamed $(7.4 \pm 5.1 \mathrm{nmol} ; P=0.0025)$.

\section{Discussion}

The new method for CHX determination fulfilled all requirements for bioanalytical measurements as described by [35, 34]. All parameters checked, such as intra- and inter-assay coefficients of variance as well as the recovery rate and the lower limit of quantification, proved the method being suitable for detection of low CHX-concentrations in small sample volumes. The LLOQ calculated was in a range of about $1.0 \mathrm{nmol} /$ well. Thus, the LLOQ fell below the CHX release per day measured in a pilot study for samples treated

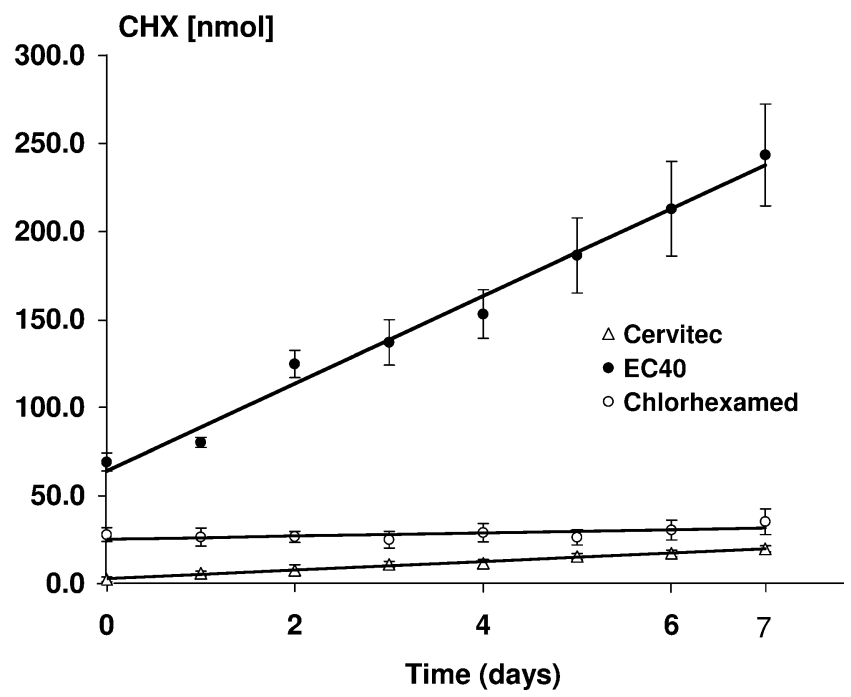

Fig. 5 Cumulative mean (and SD) of net CHX (nmol) recorded in the storage solutions containing the enamel samples either treated with Cervitec, EC40, or Chlorhexamed. The CHX determined in the storage solution is given for each day of the 7-day-period of storage in the same solution. A regression line is drawn for each CHX application with the CHX gel Chlorhexamed. To increase the amount of CHX in the storage solutions, we, therefore, had decided to measure the CHX release in the same solution during the 7-day experimental period. This means that the CHXtreated samples were not transferred to a fresh solution for measuring daily release, as done in studies determining release of other substances from dental materials, such as fluoride release from glass ionomer cements [6, 14, 24, 29]. The same procedure of measuring CHX in the identical solution during storage was done by Huizinga et al. [20], when determining CHX release from Cervitec during 16 weeks. Due to this necessity, no daily release of CHX could be determined, and a cumulative release over time was determined. This renders it nearly impossible to estimate the CHX release from the enamel samples with respect to antimicrobial potential in the in vivo situation. Additionally, for the situation in the oral cavity, it has to be considered that $\mathrm{CHX}$, which is released from a carrier, is diluted or bound to various surfaces and might interact with salivary proteins and salivary bacteria. Nevertheless, in the present study, the cumulative release for all forms of CHX application was above the minimal inhibitory concentration (MIC) of CHX, which is given as 0.19$2.0 \mu \mathrm{g} / \mathrm{ml}$ [17]. Taking the molar weight of 506 for CHX, the MIC would equal $0.26-2.76 \mathrm{nmols}$ in our storage solution volume of $0.7 \mathrm{ml}$. This value was exceeded by both the varnishes and the gel at least directly after application. Even the approximate daily release from the two varnishes was above this calculated MIC. Nevertheless, extrapolation of the obtained in vitro data to the clinical situation with respect to possible bacterial inhibition should be done with caution.

The results of the study showed clearly that the two CHX varnishes tested have the potential to release measurable amounts of CHX after application to an artificial fissure system which is brushed regularly. Furthermore, after application of the Chlorhexamed gel, a constant although lower release as compared to the varnishes was recorded. The higher CHX release from the varnish EC40 as compared to Cervitec might be attributed to the higher concentration of the EC40. This becomes obvious when referring to the CHX release immediately after application. However, also in the period after day 1, the daily release was higher from EC40 represented by the steeper slope of the regression line of EC40 as compared to Cervitec (Fig. 5). Nevertheless, in contrast to the CHX gel, also Cervitec showed retention in the fissure system under the simulated toothbrushing procedure resulting in a prolonged and steady CHX release after application of the varnish onto the fissures. The better retention of EC40 in contrast to Cervitec might be attributed to the higher stickiness of EC40 and the fact that EC40 hardens to a solid consistency difficult to remove from the fissures by toothbrushing. 
The study is the first study measuring CHX release after application onto a fissure system. The extensions of the fissures were prepared as previously done by Smits and Arends [36] in an in vitro study. Use of artificial fissures was necessary in our study to standardize the determination of CHX release. However, it should be noted that natural fissures might have various shapes, thus allowing different retention of a varnish applied and different access of toothbrush bristles. This means that retention of the tested CHX regimes to natural fissures might, in some part, be different as compared to the present in vitro situation.

The present study was limited to a period of 7 days. For a better understanding of the CHX action in the oral cavity, a longer observation period would be desirable. In a future study, it should be clarified how long the two varnishes tested are able to show a measurable release of CHX. However, antimicrobial therapies utilizing CHX for reducing caries risk are mostly limited to a certain period, ranging from a single application of a highly concentrated product to multiple applications of lower concentrated gels during this treatment period $[7,10,15,22$, 43]. These restricted periods seem to be sufficient to reduce the critical mass of carious-inducing bacteria for a certain period of time, as shown in numerous previous studies. Thus, it could be assumed that the CHX release as observed in the present study for 7 days could contribute to reducing the carious risk in fissures. Nevertheless, repeated applications after a certain period of time of at least 6 months seem to be advisable [26].

In conclusion, the study proved the newly introduced method as a reliable tool to detect and quantify minimal CHX contents in small volumes. Additionally, under the chosen conditions, both tested varnishes demonstrated prolonged and higher CHX release from artificial fissures in comparison to the CHX gel tested.

\section{References}

1. Achong RA, Briskie DM, Hildebrandt GH, Feigal RJ, Loesche WJ (1999) Effect of chlorhexidine varnish mouthguards on the levels of selected oral microorganisms in pediatric patients. Pediatr Dent 21:169-175

2. Andermann G, Buhler MO, Erhart M (1980) Rapid colorimetric analysis of chlorhexidine in pharmaceutical preparations. J Pharm Sci 69:215-217

3. Araujo AM, Naspitz GM, Chelotti A, Cai S (2002) Effect of Cervitec on mutans streptococci in plaque and on caries formation on occlusal fissures of erupting permanent molars. Caries Res 36:373-376

4. Attin R, Ilse A, Werner C, Wiegand A, Attin T (2006) Antimicrobial effectiveness of a highly concentrated chlorhexidine varnish treatment in teenagers with fixed orthodontic appliances. Angle Orthod 76:1022-1027

5. Attin R, Tuna A, Attin T, Brunner E, Noack MJ (2003) Efficacy of differently concentrated chlorhexidine varnishes in decreasing
Mutans streptococci and lactobacilli counts. Arch Oral Biol 48:503-509

6. Attin T, Buchalla W, Ameling K, Hellwig E (1999) Effect of toothbrushing on fluoride release of polyacid-modified composite resins. Clin Oral Invest 3:150-154

7. Baca P, Munoz MJ, Bravo M, Junco P, Baca AP (2002) Effectiveness of chlorhexidine-thymol varnish for caries reduction in permanent first molars of 6-7-year-old children: 24-month clinical trial. Community Dent Oral Epidemiol 30:363-368

8. Baca P, Munoz MJ, Bravo M, Junco P, Baca AP (2004) Effectiveness of chlorhexidine-thymol varnish in preventing caries lesions in primary molars. J Dent Child (Chic) 71:61-65

9. Brambilla E, Cagetti MG, Fadini L, Pariset P, Strohmenger L, Twetman S (2004) Chlorhexidine concentration in saliva after topical treatment with an antibacterial dental varnish. Am J Dent 17:196-198

10. Bratthall D, Serinirach R, Rapisuwon S, Kuratana M, Luangjarmekorn V, Luksila K, Chaipanich P (1995) A study into the prevention of fissure caries using an antimicrobial varnish. Int Dent J 45:245-254

11. Brougham LR, Cheng HY, Pittman KA (1986) Sensitive highperformance liquid chromatographic method for the determination of chlorhexidine in human serum and urine. J Chromatogr 383:365-373

12. Cabral CT, Fernandes MH (2007) In vitro comparison of chlorhexidine and providone-iodine on the long-term proliferation and functional activity of human alveolar bone cells. Clin Oral Invest 11:155-164

13. de Vries J, Ruben J, Arends J (1991) Determination of chlorhexidine in saliva and in aqueous solutions. Caries Res 25:410-414

14. Delbem AC, Pedrini D, Franca JG, Machado TM (2005) Fluoride release/recharge from restorative materials-effect of fluoride gels and time. Oper Dent 30:690-695

15. Fennis-le YL, Verdonschot EH, Burgersdijk RC, Konig KG, van't Hof MA (1998) Effect of 6-monthly applications of chlorhexidine varnish on incidence of occlusal caries in permanent molars: a 3-year study. J Dent 26:233-238

16. Gjermo P (1989) Chlorhexidine and related compounds. J Dent Res 68:1602-1608

17. Hennessey TS (1973) Some antibacterial properties of chlorhexidine. J Periodontal Res 12:61-67

18. Holbrook A (1958) The determination of small quantities of chlorhexidine in pharmaceutical preparations. J Pharm Pharmacol 10:370-374

19. Hoszek A, Struzycka I, Jozefowicz A, Wojcieszek D, Wierzbicka M, Wretlind K, Ericson D (2005) Chlorhexidine-containing glass ionomer cement. A clinical investigation on the fissure caries inhibiting effect in first permanent molars. Swed Dent J 29:89-96

20. Huizinga ED, Ruben JL, Arends J (1991) Chlorhexidine and thymol release from a varnish system. J Biol Buccale 19:343-348

21. Imfeld $T$ (2006) Chlorhexidine-containing chewing gum. Clinical documentation. Schweiz Monatsschr Zahnmed 116:476-483

22. Jenatschke F, Elsenberger E, Welte HD, Schlagenhauf U (2001) Influence of repeated chlorhexidine varnish applications on mutans streptococci counts and caries increment in patients treated with fixed orthodontic appliances. J Orofac Orthop 62:36-45

23. Joharji RM, Adenubi JO (2001) Prevention of pit and fissure caries using an antimicrobial varnish: 9 month clinical evaluation. J Dent 29:247-254

24. Kuvvetli SS, Tuna EB, Cildir SK, Sandalli N, Gencay K (2006) Evaluation of the fluoride release from orthodontic band cements. Am J Dent 19:275-278

25. Lorenz K, Bruhn G, Heumann C, Netuschil L, Brecx M, Hoffmann T (2006) Effect of two new chlorhexidine mouthrinses on the development of dental plaque, gingivitis, and discolouration. A randomized, investigator-blind, placebo-controlled, 3-week experimental gingivitis study. J Clin Periodontol 33:561-567 
26. Matthijs S, Adriaens PA (2002) Chlorhexidine varnishes: a review. J Clin Periodontol 29:1-8

27. Mizrak T, Guncu GN, Caglayan F, Balci TA, Aktar GS, Ipek F (2006) Effect of a controlled-release chlorhexidine chip on clinical and microbiological parameters and prostaglandin E2 levels in gingival crevicular fluid. J Periodontol 77:437-443

28. Pacheco-Fowler V, Gaonkar T, Wyer PC, Modak S (2004) Antiseptic impregnated endotracheal tubes for the prevention of bacterial colonization. J Hosp Infect 57:170-174

29. Pamir T, Tezel H, Ozata F, Celik A (2006) Fluoride release from esthetic restorative materials with and without adhesive. Quintessence Int 37:145-150

30. Pinzauti S, La Porta E, Casini M, Betti C (1982) Colourimetric assay of chlorhexidine in contact lens solutions using methyl orange. Pharm Acta Helv 57:334-337

31. Rosenthal S, Spangberg L, Safavi K (2004) Chlorhexidine substantivity in root canal dentin. Oral Surg Oral Med Oral Pathol Oral Radiol Endod 98:488-492

32. Rozier RG (2001) Effectiveness of methods used by dental professionals for the primary prevention of dental caries. J Dent Educ 65:1063-1072

33. Schwarz F, Bieling K, Bonsmann M, Latz T, Becker J (2006) Nonsurgical treatment of moderate and advanced periimplantitis lesions: a controlled clinical study. Clin Oral Invest 10:279-288

34. Shah VP, Midha KK, Dighe S, McGilveray IJ, Skelly JP, Yacobi A, Layloff T, Viswanathan CT, Cook CE, McDowall RD (1991) Analytical methods validation: bioavailability, bioequivalence and pharmacokinetic studies. Conference report. Eur J Drug Metab Pharmacokinet 16:249-255
35. Shah VP, Midha KK, Findlay JW, Hill HM, Hulse JD, McGilveray IJ, McKay G, Miller KJ, Patnaik RN, Powell ML, Tonelli A, Viswanathan CT, Yacobi A (2000) Bioanalytical method validation - a revisit with a decade of progress. Pharm Res 17:1551-1557

36. Smits MT, Arends J (1988) Influence of extraoral xylitol and sucrose dippings on enamel demineralization in vivo. Caries Res 22:160-165

37. Stevens LE, Durrwachter JR, Helton DO (1986) Analysis of chlorhexidine sorption in soft contact lenses by catalytic oxidation of [14C]chlorhexidine and by liquid chromatography. J Pharm Sci $75: 83-86$

38. Twetman S (2003) Evidence for caries prevention by chlorhexidine varnish in schoolchildren and adolecents at caries risk. Oralprophylaxe 25:41-44

39. Twetman S (2004) Antimicrobials in future caries control? A review with special reference to chlorhexidine treatment. Caries Res 38:223-229

40. Weston DJ, Bateman R, Wilson ID, Wood TR, Creaser CS (2005) Direct analysis of pharmaceutical drug formulations using ion mobility spectrometry/quadrupole-time-of-flight mass spectrometry combined with desorption electrospray ionization. Anal Chem 77:7572-7580

41. Wiegand A, Otto YA, Attin T (2004) In vitro evaluation of toothbrushing abrasion of differently bleached bovine enamel. Am J Dent 17:412-416

42. Wilson SJ, Wilson HJ (1993) A method for the estimation of traces of chlorhexidine in water. J Oral Rehabil 20:225-231

43. Zickert I, Lindvall AM, Axelsson P (1982) Effect on caries and gingivitis of a preventive program based on oral hygiene measures and fluoride application. Community Dent Oral Epidemiol 10:289-295 